\title{
The role of EEG recordings in children undergoing cardiac surgery for congenital heart disease
}

\author{
Sascha Meyer ${ }^{\mathrm{a}, *, 1}$, Mohammed Ghiath Shamdeen ${ }^{\mathrm{a}, 1}$, Mohammed Shatat ${ }^{\mathrm{b}}$, Hans-Jochaim Schäfers ${ }^{\mathrm{c}}$, \\ Ludwig Gortner ${ }^{\mathrm{a}}$, Sven Gottschling ${ }^{\mathrm{d}}$ and Hashim Abdul-Khaliq ${ }^{\mathrm{b}}$ \\ ${ }^{a}$ University Hospital of Saarland, Department of Pediatrics and Neonatology, Neuropediatrics, Homburg/Saar, Germany \\ ${ }^{\mathrm{b}}$ Department of Pediatric Cardiology, University Hospital of Saarland, Germany \\ ${ }^{\mathrm{c}}$ Department of Cardiothoracic Surgery, University Hospital of Saarland, Germany \\ ${ }^{\mathrm{d}}$ Department of Pediatrics, University Hospital of Saarland, Germany
}

\begin{abstract}
Neurological dysfunction may occur after corrective cardiac surgery using cardio-pulmonary bypass (CPB) with or without circulatory arrest. Different neurophysiological monitoring systems have been employed to detect neurological complications and possible brain injury in infants and children during and after cardiac surgery. The value of EEG in infants and children at risk for neurological sequelae has not been systematically studied.

Sequential performance of 2 EEGs before and after cardiac surgery at a tertiary University Hospital to screen for possible brain injury after cardiac surgery. In addition, a complete neurological examination was performed.

In 313 patients (age: $54.2 \pm 55.7$ months; normal initial EEG) after cardiac surgery CPB (duration of surgery: $146.0 \pm 58.9$ minutes; aortic cross clamp time: $34.1 \pm 19.1$ minutes) a 19-channel EEG recording was performed $2.4 \pm 1.8$ days prior and $11.6 \pm 5.3$ days after cardiac surgery. An abnormal EEG was detected in only 8 of 313 patients (2.5\%; focal slowing(1), generalised slowing (5), epiletiform discharges(2)) after cardiac surgery, while the EEG was normal in the remaining 305 patients (97.5\%). In one patient, an intra-cerebral pathology was seen on MRI (ischemic); in 5 patients follow-up EEGs were performed, which revealed normalised findings. None of the 8 patients demonstrated new focal neurological deficits on physical examination, but $33(9.7 \%)$ children demonstrated minor abnormalities (eg, subtle motor asymmetry, increase in muscle tone, etc.), which were unrelated to abnormal EEG findings.

According to the used protocol pathological EEG findings were very infrequent in our study cohort. The routine and indiscriminative recording of EEGs in children before and after corrective or palliative cardiac surgery for congenital heart disease using $\mathrm{CPB}$ is not recommended. Further intra-operative neuromonitoring methods with immediate intervention should be evaluated.
\end{abstract}

Keywords: Congenital heart disease, cardiac surgery, cardiopulmonary bypass, electroencephalography, children, neurological sequelae

\section{Introduction}

Neurological and psychomotor deficits still occur in pediatric patients after surgery of congenital heart disease with cardiopulmonary bypass (CPB) with or

*Corresponding author: Sascha Meyer, University Hospital of Saarland, Department of Pediatrics and Neonatology, Neuropediatrics Building 9, Kirrbergerstr., 66421 Homburg/Saar, Germany ${ }^{1}$. Tel.: +49 06841 1628374; E-mail: sascha.meyer@uniklinik-saarland.de.

${ }^{1} \mathrm{SM}$ and MGS contributed equally to this work without periods of deep hypothermic circulatory arrest $[1,2]$. The etiology of cerebral injury associated with cardiac surgery in neonates and infants is considered as multifactor and has not been clearly elucidated [1,3-5]. Visible brain lesions such as intracranial hemorrhages and non-visible lesions resulting in long-term neuropsychological and cognitive deficits have been reported in association with congenital heart malformations and corrective cardiac surgery [6-9]. However, the precise etiology and time course both global or focal brain injury remains a matter of debate. In addition to possible 
congenital brain abnormalities a causal relationship has been suggested between the neurological sequelae and several non-physiological factors during corrective cardiac surgery such as variations in blood flow, oxygenation, temperature and acid-base status [10,11]. Postoperatively hyperventilation, low cardiac output sydrome, and variation in intravascular volume during the early postoperative period contribute to alterations in cerebral hemodynamics and thereby to the possible development of cerebrovascular injury $[12,13]$. Several cerebral imaging modalities may be used to detect abnormal intracranial findings before and after surgery. However, these methods are not routinely used in all neurologically asymptomatic infants and children undergoing cardiac surgery. Certainly, in neonates and infants transcranial ultrasound is used routinely to detect possible intraventricular or periventricular hemorrhage.

A strong predictive value of adverse neurological and developmental outcome at 1 year of age was found in those infants who had evidence of seizure activity during the first 48 postoperative hours after corrective cardiac surgery [14]. The value of using routine EEG to assess abnormal cerebral activity before and after surgery in large groups of patients undergoing corrective or palliative cardiac surgery has not been formally reported as of now.

The aim of this study was to assess the role of routine EEG recordings prior and after cardiac surgery to detect neurophysiological abnormalities, and to relate the EEG findings to clinical findings on neurological examination.

\section{Patients and methods}

This study was done in accordance with the policy of the Institutional Review Board and Ethics Committee of the University Hospital of Saarland, Homburg, Germany. Parental consent was obtained prior to enrolment.

All patients with planned/elective corrective or palliative cardiac surgery using CPB in our department were enrolled in this study, and their data (age, gender, type of cardiac lesion, type and length of surgery, time of aortic cross clamp, results of EEG studies, and results of neurological examination) were retrieved from an electronic database. The recording of EEGs before and after cardiac surgery was routine practice in our hospital.

Enrolment criteria: Patients (aged 0-17 years) admitted to our hospital for planned corrective/palliative cardiac surgery. All data were stored using SPSS 17.0 software. Data are presented as mean \pm standard deviation.

\section{EEG recordings}

A 19-channel EEG recording was performed prior to cardiac surgery and before PICU/hospital discharge. Only children with a normal initial EEG were included in this study. In addition, a complete neurological examination was performed before discharge.

Twenty silver cup electrodes were placed according to the 10-20 international system. Electrode impedances measured less than $5 \mathrm{kOhm}$. An IT med ${ }^{\circledR}$ (IT Medical, Usingen, Germany) model EEG Neurofile NT/XP machine was used to record 12 channels. A high-frequency channel was set at $70 \mathrm{~Hz}$; bipolar longitudinal and transversal montages were used. Each EEG recording lasted 15-20 min. All EEGs were evaluated by the same experienced neuropediatrician (MGS), based on conventional EEG criteria. Age-dependent EEG differences were taken into account [15].

Abnormal EEG findings were divided into 3 categories: a) focal slowing b) generalised slowing c) epileptiform discharges (Fig. 1a-c).

Prior to discharge and after recording of the second EEG a formal clinical neurological clinical examination was performed to look for specific neurological deficits. We did not assess cognitive function before discharge. Also, we did not routinely perform other studies to search for neurological abnormalities (biomarkers, imaging studies, etc.).

\section{Results}

Between January 2004 and January 2008 a total of 313 patients (mean age: $54.2 \pm 55.7$ months) undergoing cardiac surgery with CPB (duration of surgery: 146.0 \pm 58.9 minutes; aortic cross clamp time: $34.1 \pm 19.1 \mathrm{~min}-$ utes) were included in this study (58 ASD; 72 VSD, 33 Fallot, 51 valve anomalies; 19 TGA, 15 AV canal, 65 other complex anomalies including single ventricle etc.).

Surgical procedures included a variety of procedures, eg, closure of atrio-septal defects, ventricular septal defects, arterial switch surgery for transposition of the great arteries, Norwood stage I surgery, HemiFontan, Fontan, Glenn procedure, Damus-Kaye-Stensel surgery, and pulmonary and aortic valve replacement or reconstruction.

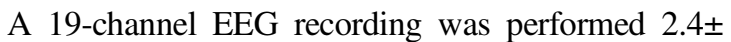
1.8 days prior and $11.6 \pm 5.3$ days after cardiac surgery. An abnormal EEG was detected in only 8 of 313 patients (2.5\%; focal slowing (1), generalised slowing (5), epiletiform discharges (2)) after cardiac surgery, while the EEG was normal in the remaining 305 patients 
(97.5\%). In one patient, an intra-cerebral pathology was seen on MRI (ischemic lesion); in 5 patients follow-up EEGs were performed, which revealed normalised findings. Three patients did not present for the recording of a follow-up EEG. No significant differences between children with EEG abnormalities and normal EEGs were seen with regard to underlying disease, type and length of surgery, and aorticcross clamp time. None of the 8 patients demonstrated new neurological abnormalities or deficits on physical examination; however, 33 $(9.7 \%)$ children demonstrated minor abnormalities (eg, subtle motor asymmetry, increase in muscle tone, etc.), which were unrelated to abnormal EEG findings. Again, this group was not significantly from children without minor neurological abnormalities with regard to underlying disease, type and length of surgery, and aorticcross clamp time.

The occurrence of an abnormal EEG was not associated with a specific type of surgical procedure nor was it linked to CPB or aortic cross clamp time.

On neurological examination, we did not detect any new focal/specific neurological deficits in any patient that was related to the recent cardiac surgery. None one the patients developed overt clinical seizure activity. As we did not perform neuropsychological testing, we could not assess neuro-cognitive function prior to discharge. Also, we could not rule very subtle neurological deficits.

\section{Discussion}

Electrophysiological techniques are among the most frequently used methods to provide information about the functioning of the human brain [16]. These techniques are useful in that they are non-invasive and relatively inexpensive. The main result of our study demonstrated a low incidence of abnormal EEG findings after surgery in pediatric patients undergoing heart surgery for congenital heart disease. Of interest, in none of our patients did we detect focal or generalized neurological deficits. This can be attributed to the fact that the routine use of $\mathrm{CPB}$ during pediatric cardiac surgery has been optimized and is almost completely safe in all mild and complex surgical procedure.

Although, the conducted EEG monitoring before and after cardiac surgery may not necessarily reflect the cerebral monitoring of critical period during and immediately after CPB, we did not detect overt focal neurological deficits in the studied patients, thus giving evidence to no new focal or global cerebral ischemic events. Of note, only few studies have assessed the role of intra-operative EEG monitoring in children undergoing open-heart surgery.

A very recent study by Andopoulos et al. assessed the occurrence of electroencephalographic seizures after neonatal cardiac surgery with high-flow "cardiopulmonary bypass" [17]. In their study, the authors demonstrated that EEG seizures are infrequent (1.5\%) in neonates undergoing surgery with high-flow CPB, irrespective of single ventricle or 2-ventricle underlying congenital heart disease. This is to some degree comparable to our findings. Given the low incidence, the authors question the utility of EEG seizures as a marker of acute brain injury, and also as a potential surrogate parameter for long-termneurological outcome [17]. This is an important finding as seizure activity during the first 48 postoperative hours after corrective cardiac surgery has been postulated to be a strong predictive value of adverse neurological and developmental outcome at 1 year of age [14].

Our study was not aimed to monitor the brain physiology during CPB or immediately after surgery, when the patients were under sedation, but rather to screen for possible brain events before discharge. Intra-operatively the standard EEG with 12 channels has been used to monitor brain electrical activities during and after heart surgery in congenital heart surgery with contradictory results $[18,19]$. In the study by Austin et al., EEG, transcranial Doppler ultrasonic measurement of middle cerebral artery blood flow velocity, and transcranial near-infrared cerebral oximetry were monitored in 250 patients, and an intervention algorhythm was developed to detect and correct specific deficiencies in cerebral perfusion or oxygenation or to increase cerebral tolerance to ischemia or hypoxia. From their experience the authors concluded that interventions based on neurophysiologic monitoring appeared to decrease the incidence of postoperative neurologic sequelae and reduce the length of stay [18]. Conversely, Miller et al. analyzed the intraoperative EEG changes during open heart surgery with deep hypothermia in 66 infants aged 6 months or younger, $70 \%$ of whom were neonates in a prospective study [19]. The authors compared suppression of amplitude and continuity at the nadir of temperature reduction and following rewarming, and the appearance of periodic paroxysmal activity with neurologic abnormalities before and following operation, patient characteristics, and operation variables. Of interest, EEG changes did not disclose any relationship to abnormal neurologic findings, age at operation, type of anesthetic, duration of $\mathrm{CPB}$, duration of low-flow $\mathrm{CPB}$ or cooling, temperature at circulatory arrest or low flow, or nasopharyngeal-venous return temperature differences. 
The routine recording of an EEG during cardiac surgery is challenging because of interference of the registered signals by several factors during CPB such as, sedative and analgesic medications, hypothermia and re-warming, variation of cerebral perfusion on CPB, and deep hypothermic circulatory arrest. The complexity to place the electrodes in right and stable position during such major heart operations may represent an additional limitation to use the methods for routine brain monitoring during cardiac surgery.

Other methods for cerebral monitoring during and after cardiac surgery have emerged in recent years, including NIRS, sonographic and ultrasound studies as well as the use of biochemical markers (eg, neuronspecific enolase, S100B protein [20]. However, the role of these new modalities in these complex clinical settings has not yet been fully established [21-23].

In summary, our study demonstrated that the studied infants and children did not develop new focal neurological deficits after cardiac surgery according to basic clinical/neurological assessment and EEG monitoring, although s substantial percentage of children developed minor abnormalities when assessed by a physiotherapist. These findings were unrelated to abnormal EEG findings.

We conclude that the long term effect of corrective cardiac surgery and possible altered psychomotor development in association with congenital heart defects should be evaluated using other clinical test. A shortcoming of our study was the fact that we did not formally perform neuro-cognitive functioning tests (eg, Kaufmann-ABC) to detect intellectual deficits secondary to cardiac surgery. Moreover, subtle changes/deficits in their neurological performance may have not been noticed. Of note, most of the included patients were elective or semielective patients, and we could not include emergency patients (as the "routine" recording of a baseline EEG is not feasible in this cohort of patients) who are at higher risk of developing neurological dysfunction. Also, we only recorded one follow-up EEG after cardiac surgery. Thus, the true incidence of post-operative EEG abnormalities including seizure activity may be under-estimated in our study.

We conclude that routine EEG monitoring may not be indicated in children undergoing cardiac surgery. An individual approach for children with a high risk for neurological complications and long-term sequelae may be more promising. Also, there is a considerable body of experimental work suggesting that more complex EEG paradigms may one day be used to assess changes in brain function after injury (discriminating functions based on patterns of coherence, phase, and amplitude) [24]. A major limitation of this study was that EEG monitoring was not performed immediately during, and more importantly after CPB to detect possible post perfusion seizures, which have strongly associated with long-term neurological sequelae [14].

\section{Appendix}

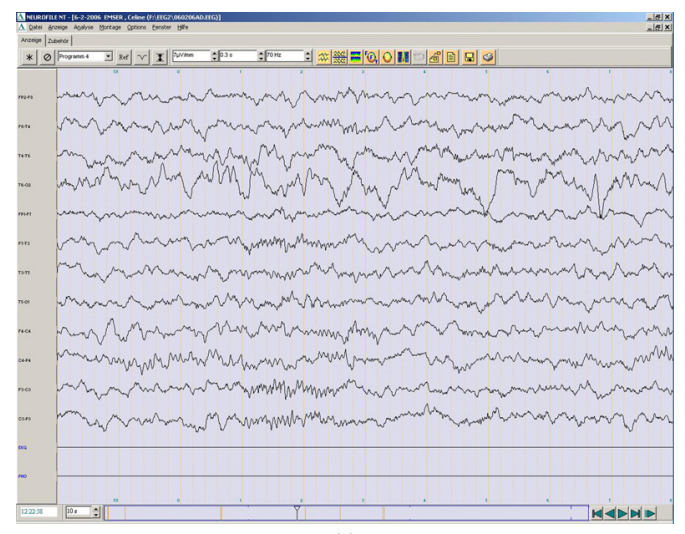

(a)

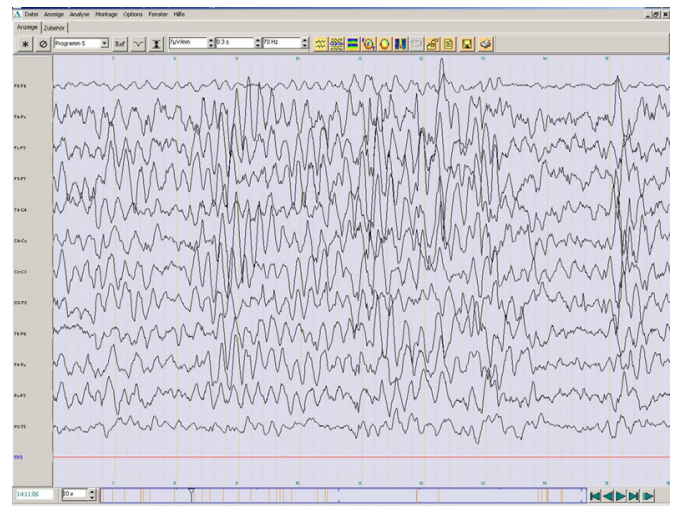

(b)

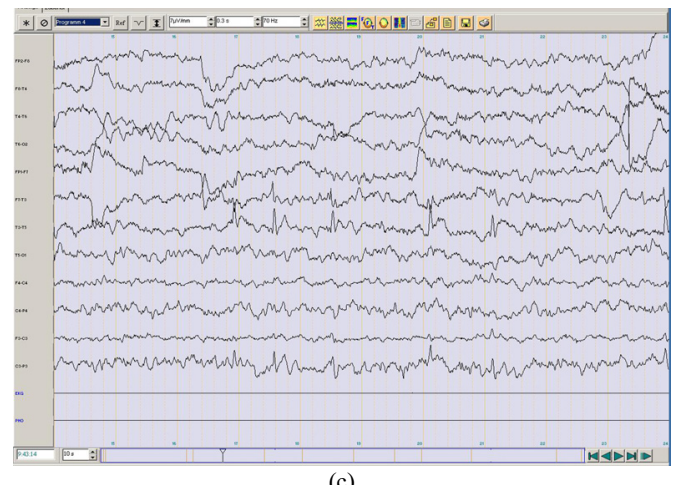

(c)

Fig. 1. Pathological EEG recordings. a) Focal slowing in right temporal occipital lead, b) Generalised slowing, c) Epileptiform discharges (Spikes-and-wave complexes). 


\section{Authors' contributions}

Sascha Meyer, Mohammed Ghiath Shamdeen, Ludwig Gortner, and Hashim Abdul-Khaliq were responsible for the study design, data analysis and writing of the manuscript.

Mohammed Shatat and Sven Gottschling were responsible for data aquisition and data analysis.

Hans-Joachim Schäfers was responsible for patient treatment, and writing of the manuscript.

\section{References}

[1] Newburger JW, Jonas RA, Wernovsky G, Wypij D, Hickey $\mathrm{PR}$, Kuban KC, et al. A comparison of the perioperative neurologic effects of hypothermic circulatory arrest versus lowflow cardiopulmonary bypass in infant heart surgery. N Engl J Med 1993; 329: 1057-64.

[2] Bellinger DC, Jonas RA, Rappaport LA, Wypij D, Wernovsky $\mathrm{G}$, Kuban KC, et al. Developmental and neurologic status of children after heart surgery with hypothermic circulatory arrest or low-flow cardiopulmonary bypass. N Engl J Med 1995; 332: 549-5.

[3] Ferry PC. Neurologic sequelae of open-heart surgery in children. An 'irritating question'. Am J Dis Child 1990; 144(3): 369-73.

[4] Ferry PC. Neurologic sequelae of cardiac surgery in children. Am J Dis Child 1987; 141: 309-12.

[5] Cooper W, Duarte I, Thourani V, Nakamura M, Wang N, Brown M, et al. Hypothermic circulatory arrest causes multisystem vascular endothelial dysfunction and apoptosis. Ann Thorac Surg 2000; 69: 696-703.

[6] Miller G, Vogel H. Structural evidence of injury or malformation in the brains of children with congenital heart disease. Semin Pediatr Neurol 1999; 6: 20-6.

[7] Keck EW, Kimm E, Grävinghoff L, Sieg K, Lagenstein I, Kühne D. Neurologische Veränderung und zerebrale Läsionen bei Kindern mit Transposition der großen Arterien (TGA). Monatsschr Kinderheilkd 1981; 129: 45-7.

[8] Van Houten JP, Rothman A, Bejar R. High incidence of cranial ultrasound abnormalities in full-term infants with congemital heart disease. Am J Perinatol 1996; 13: 47-53.

[9] Bozoky B, Bara D, Kertesz E. Autopsy study of cerebral complications of congenital heart disease and cardiac surgery. J Neurol 1984; 231(3): 153-61.

[10] Terplan KL. Brain changes in newborns, infants and children with congenital heart disease in association with cardiac surgery. Additional observations. J Neurol 1976; 212(3): 225-36.
[11] Greeley WJ, Kern FH, Mault JR, Ungerleider R. Mechanism of injury and methods of protection, during cardiac surgery in neonates and infants. Cardiol Young 1993; 3: 317-30.

[12] Greeley WJ, Ungerleider RM, Kern FH, Brusino FG, Smith R, Revers JG. Effects of cardiopulmonary bypass on cerebral blood flow in neonates, infants, and children. Circulation 1989; 80(Suppl. 1): 209-15.

[13] Jonasson AE, Quaegebeur J, Young LY. Cerebral blood flow velocity in pediatric patients is reduced after cardiopulmonary bypass with profound hypothermia. J Thorac Cardiovasc Surg 1995; 110: 934-43.

[14] Rappaport LA, Wypij D, Bellinger DC, Helmers SL, Holmes GL, Barnes PD, et al. Relation of seizures after cardiac surgery in early infancy to neurodevelopmental outcome. Boston Circulatory Arrest Study. Circulation 1998; 97: 773-9.

[15] Niedermeyer E. Maturation of the EEG: development of waking and sleep patterns. In: Niedermeyer E, Lopes Da Silva F, editors. Electroencephalography. Baltimore: Williams \& Wilkens; 1993. p. 167-91.

[16] Jacobson M, Sperling MR. The electroencephalogram in minor brain injury. In: Mandel S, Sataloff RT, Schapiro SR, editors. Minor head trauma: assessment, management, and rehabilitation. New York: Springer-Verlag; 1993.

[17] Andropoulos DB, Mizrahi EM, Hrachovy RA, Stayer SA, Stark AR, Heinle JS, et al. Electroencephalographic seizures after neonatal cardiac surgery with high-flow cardiopulmonary bypass. Anaesth Analg 2010; 110: 1680-5.

[18] Austin III EH, Edmonds Jr HL, Auden SM, Seremet V, Niznik G, Sehic A, et al. Benefit of neurophysiologic monitoring for pediatric cardiac surgery. J Thorac Cardiovasc Surg 1997; 114: 707-15.

[19] Miller G, Rodichol LD, Baylen BG, Myers JL. EEG changes during open heart surgery on infants aged 6 months or less. Pediatr Neurol 1994; 10: 124-30.

[20] Abdul-Khaliq H, Schubert S, Stoltenburg-Didinger G, Troitzsch D, Bottcher W, Hubler M, et al. Protein S-100beta in brain and serum after deep hypothermic circulatory arrest in rabbits: relationship to perivascular astrocytic swelling. Clin Chem Lab Med. 2000; 38: 1169-72.

[21] Meyer S, Gottschling S, Baghai A, Polcher T, Strittmatter M, Gortner L. The role of S100B-protein in neonatology, pediatric intensive care, and pediatrics. Klin Padiatr 2006; 218: 49-56.

[22] Abdul-Khaliq H, Troitzsch D, Schubert S, Wehsack A, Böttcher W, Gutsch E, et al. Cerebral oxygen monitoring during neonatal cardiopulmonary bypass and deep hypothermic circulatory arrest. Thorac Cardiovasc Surg 2002; 50: 77-81.

[23] Kunihara T, Tscholl D, Langer F, Heinz G, Sata F. Cognitive brain function after hypothermic circulatory arrest assessed by cognitive P300 evoked potentials. Eur J Cardiothorac Surg 2007; 32: 507-13.

[24] Thatcher RW, Walker RA, Gerson I, Geiser FH. EEG discriminant analyses of mild head trauma. Electroencephalogr Clin Neurophysiol 1989; 73: 94-106. 